\title{
openheart Direct oral anticoagulants versus warfarin: is new always better than the old?
}

\author{
John Burn, ${ }^{1}$ Munir Pirmohamed ${ }^{2}$
}

To cite: Burn J, Pirmohamed M. Direct oral anticoagulants versus warfarin: is new always better than the old?. Open Heart 2018;5:e000712. doi:10.1136/ openhrt-2017-000712

Received 6 September 2017 Revised 12 December 2017 Accepted 29 December 2017
Check for updates

${ }^{1}$ Institute of Genetic Medicine, Newcastle Univerisity, Centre for Life, Newcastle upon Tyne, UK ${ }^{2}$ Department of Molecular \& Clinical Pharmacology, Institute of Translational Medicine, University of Liverpool, Liverpool, UK

Correspondence to Dr John Burn; john.burn@ newcastle.ac.uk

\section{ABSTRACT}

About 1.4 British million people are at risk of strokes due to non-valvular atrial fibrillation (AF) necessitating long-term anticoagulation. The vitamin $\mathrm{K}$ antagonist, warfarin, has a long half-life and narrow therapeutic range necessitating regular monitoring and is a common cause of iatrogenic hospital admission. Direct-acting oral anticoagulants (DOACs), dabigatran, rivaroxaban, apixaban and edoxaban are not required to have monitoring but are sensitive to changes in renal function and are associated with poorer adherence. There are good grounds to believe that DOACs are not always superior to warfarin in routine practice particularly with an older population. Much higher levels of therapeutic effectiveness can be achieved using a simple genotype guidance to identify those who are highly sensitive and by adoption of home monitoring. These adjustments could make warfarin the preferred drug for most people and would reduce the dramatic rise in health service expenditure.

It is estimated that 1.4 British million people are at risk of strokes due to non-valvular atrial fibrillation (AF) including about $4 \%$ of the over 80s. ${ }^{1}$ Antiplatelet agents are ineffective necessitating long-term anticoagulation. ${ }^{23}$ The $S$-isoform of the current standard, warfarin, works by interfering with vitamin $\mathrm{K}$ activation of clotting factors II, VII, IX and $\mathrm{X} .{ }^{4}$ Its long half-life and narrow therapeutic range necessitate regular monitoring using the international normalised ratio (INR) which needs to be held in a range of 2-3 to minimise clot formation or the converse, uncontrolled bleeding. ${ }^{5-7}$ The latter is one of the most common causes of iatrogenic hospital admission. ${ }^{8}$

Two strategies have emerged to improve the clinical utility of preventive anticoagulation: (a) the development of a new class of anticoagulants, known as the direct-acting oral anticoagulants (DOACs), including dabigatran, rivaroxaban, apixaban and edoxaban; and (b) better optimisation of warfarin use by understanding the common genetic variation in drug response and by making INR testing and dose adjustment more convenient with self-testing devices.

A series of large-scale randomised controlled trials (RCTs) published over the last five years have demonstrated apparent superiority of DOACs over warfarin for key indicators coupled with the advantage of a wider therapeutic range and a lack of a need for regular monitoring of the degree of anticoagulation. ${ }^{9-11}$ This resulted in the UK National Institute for Health and Care Excellence (NICE) guidance in 2014 that the approaches should be given equal weight at patient initiation consultations (http://nice. org.uk/guidance/cg180) and subsequently a more direct endorsement of DOACs by the European Society of Cardiology. ${ }^{12}$ As a result, there is a major shift towards DOACs in clinical practice which is placing a significant burden on health budgets. In the National Health Service (NHS) in England, expenditure on anticoagulants rose by $>£ 100$ million in $2015 / 2016$ and by around $£ 400$ million in the last financial year ${ }^{i}$. On present trends, the cost will rise to $£ 1$ billion per year by 2020 , representing $5 \%$ of the total drug budget. While the price will begin to fall in 2022 as patents expire, these costs justify a critical examination of the available evidence.

The first DOAC to be licensed, dabigatran, is a thrombin inhibitor. Some degree of interpersonal variation in response was not made clear in initial submissions leading to a prolonged legal challenge. ${ }^{13}$ A reversal agent, idarucizumab, is now licensed in the UK but is very expensive, costing $>£ 2500$ per use as opposed to parenteral vitamin $\mathrm{K}$ as a reversal agent for warfarin. ${ }^{14}$ The other three licensed agents-rivaroxaban, apixaban and edoxaban - are direct inhibitors of activated factor X. Andexanet alfa, a reversal agent of

${ }^{\mathrm{i}}$ Primary care data are from the Business Services Authority. Secondary care data are derived from the NICE 'Novel Oral Anticoagulant' Technology Assessment. 
factor Xa inhibitor activity, is under consideration to be licensed in the UK though its short half-life and high cost of $>£ 1500$ is an issue. ${ }^{15}$ The most frequently prescribed anti-Xa inhibitor, rivaroxaban, was shown to be non-inferior to warfarin in a large-scale trial which used a device for INR monitoring in the warfarin arm which was subsequently withdrawn on the US Food and Drug Administration instruction as it was unreliable. ${ }^{16}$ The original study authors concluded this had not compromised the final conclusions.

A meta-analysis of the four RCTs comparing efficacy of DOACs versus warfarin found the DOACs to be superior to warfarin in terms of embolic and haemorrhagic stroke but to be inferior in terms of gastrointestinal bleeding events. ${ }^{17}$ An important qualification was that superiority was dependent on the proportion of warfarin patients in the therapeutic window. If the average time in the therapeutic range (TTR) was $<66 \%$, the new drugs were superior but they were not superior if average TTR of patients on warfarin was $>66 \%$ (Relative Risk $=0.69,95 \%$ CI 0.59 to 0.81 vs Relative Risk $=0.93,95 \%$ CI 0.76 to 1.13 , $\left.\mathrm{P}_{\text {interaction }}=0.022\right)$.

Three other subsequent observations raise major concerns. RCTs tend to focus on younger people with fewer comorbidities. Abrahams et al ${ }^{18}$ reported in 2015 that adverse events were more common in the older population. This has a plausible biological basis since excretion rates are pivotal to maintaining correct therapeutic levels. DOACs are more dangerous in people with impaired renal function. In the frail elderly population, intercurrent illness can lead to acute decline in renal function leading to excessive anticoagulation and sometimes life-threatening bleeding. Guidance suggests that renal function should be regularly checked but compliance with this guidance is thought to be poor in practice. The importance of drug-drug interactions with DOACs is underappreciated and can occur with drugs used intermittently, for example, antibiotics such as clarithromycin. The lack of validated laboratory tests to monitor the degree of anticoagulation with DOACs means that any dose reduction in patients with impaired renal function or on interacting drugs is empirical.

The second major concern is adherence. A recent analysis (figure 1) has revealed much poorer adherence for the DOACs probably due to the lack of routine monitoring and in the case of dabigatran and apixaban, the need for twice-daily use. This analysis of adherence in England is based on a representative review of repeat prescription issuance and reveals a worrying decline in correct use over the first 12 months to just over half for rivaroxaban and apixaban and to $34 \%$ for dabigatran. These levels compare unfavourably with the $74 \%$ adherence for warfarin. Similar results have been reported in Canada. ${ }^{19}$ When the different age profile of patients in clinical practice and these new data on adherence are factored in, it becomes less likely that the DOACs will be superior to warfarin and are likely to be inferior. In those centres where TTR is $>70 \%$, these drugs are almost certainly inferior in routine practice. It is noteworthy that there is currently no systematic assessment of warfarin monitoring services. This would allow commissioners to make a more objective assessment of the current trend towards DOACs as treatment of first choice.

The third area of concern is the association between the use of DOACs and myocardial infarction. This was initially identified with dabigatran and has been confirmed by meta-analysis. ${ }^{20}$ Although initially dismissed as due to a protective effect of warfarin, rather than an

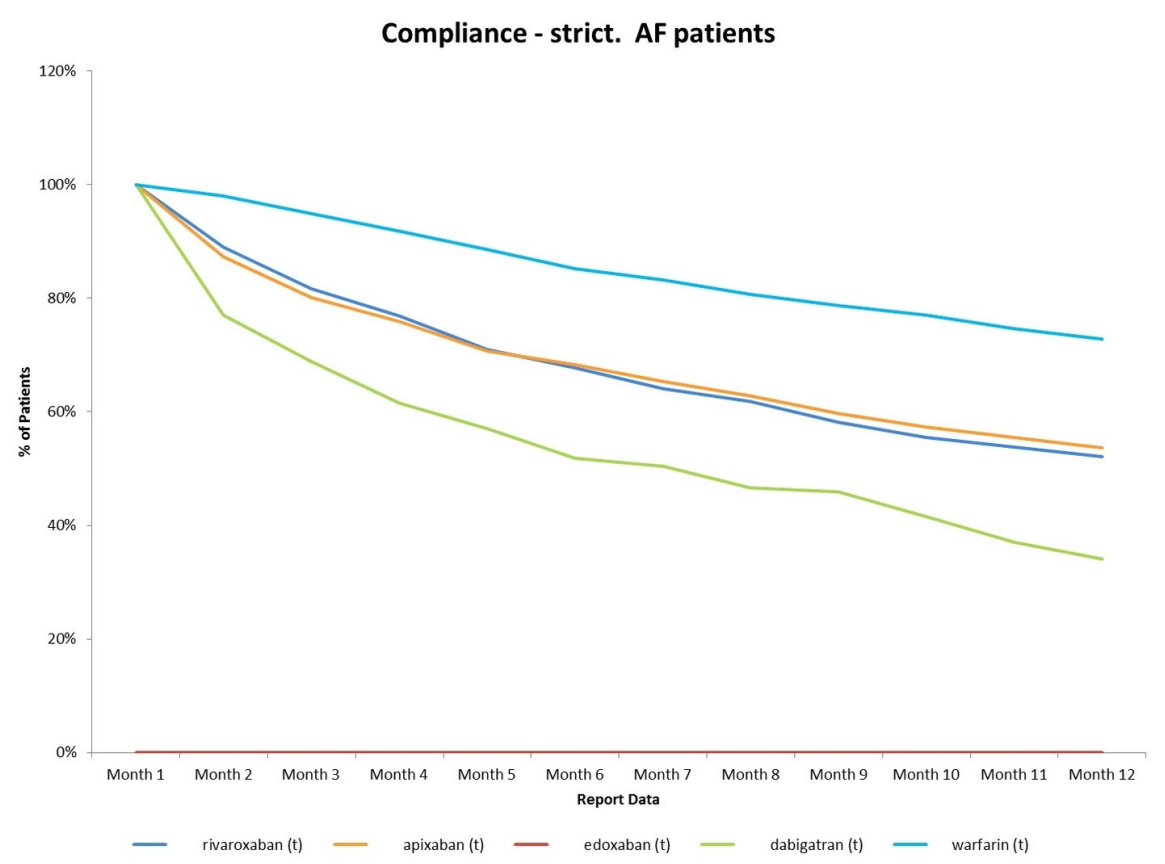

Figure 1 Adherence data for oral anticoagulants in 380 nationally distributed general practices (2143 general practitioners) in September 2016 using selected InPS Vision clinical systems. AF, atrial fibrillation. 
adverse effect of dabigatran, a recent mechanistic study has shown that dabigatran increases platelet adhesion and aggregation. ${ }^{21}$ Worryingly, more recently, it has been suggested in an observational study, ${ }^{22}$ and in a meta-analysis of randomised trials, that the anti-Xa DOACs may also increase the risk of myocardial infarction. ${ }^{23}$ Further studies are needed in this area to reduce any confounders and should include a mechanistic evaluation.

A possible tipping point in the debate has emerged recently; a systematic review of warfarin use and cancer rates using comprehensive Norwegian population-based ascertainment involving $>1.2$ million people has revealed a significant reduction in common cancers among warfarin users (Incident Rate Ratio 0.84, CI 0.82 to $0.86){ }^{24}$ Secondary analysis focused on those with AF revealed an incidence rate ratio of 0.62 (CI 0.59 to 0.65 ) with a relative risk of only 0.39 for lung cancer. In vitro studies support an anticancer effect, possibly mediated via the vitamin K-dependent product of the gene Gas6, the ligand for Axl receptor tyrosine kinase. If this observation is confirmed, the case for preferring warfarin, especially in the older population, becomes convincing.

There have been two major developments which make it possible to further improve the clinical outcomes in patients on warfarin. It has been recognised for 20 years that approximately $34 \%$ of the population carry one of two variants, known as $* 2($ rs1799853) and $* 3$ (rs1057910), in the CYP2C9 gene which is responsible for breakdown of the more active S-form of warfarin. ${ }^{25}$ People who are homozygous for either or doubly heterozygous have a much slower decline in warfarin levels and require much lower maintenance doses. Stability is harder to achieve and TTR is reduced. A second gene, VKORC1, codes for the enzyme targeted by warfarin. A common variant c.1639 G>A(rs9923231), present in 58\% of the population, reduces the expression of this gene and hence reduced levels of the enzyme, making the coagulation system more sensitive to the inhibitory effects of warfarin. ${ }^{26}$ When the declining metabolic capacity with age and the increased drug needs of the overweight are factored in, there is a 40-fold variation in weekly warfarin needs. The EU-PACT trial demonstrated that genotyping at induction allowed rapid adoption of the correct dose with a more rapid achievement of a therapeutic level and greater time in range. ${ }^{27}$ The device used in the EU-PACT trial allowed genotyping to be done in under 2 hours without significant technical expertise and is now being evaluated in a variety of near patient settings. Other devices are under development which promise even faster and easier genotyping and dose calculation. Mega et $a l^{28}$ demonstrated the impact of genotyping for CYP2C 9 and VKORC1 in a trial of edoxaban versus warfarin; in those at genotypic low risk, there was no difference in bleeding risk compared with the DOAC.

A second technical innovation has been the development of a hand-held INR testing device, the COAGuCHEK that is sufficiently simple to operate that people are able to test their INR at home and call in the result, eventually graduating in some cases to self-management of warfarin dose. A pilot study in the northeast of England has shown considerable enthusiasm among warfarin users, even those of advanced years; TTR levels of $\geq 80 \%$ have been recorded. In the NHS in England, the combined cost of warfarin prescription and annual INR monitoring, whether centrally organised and delivered or employing self-testing, is around $£ 200$ per annum. The annual prescription cost of the DOACs is in the range $£ 600-800$ to which should be added the cost of biannual renal function checks.

Although we have focused on anticoagulation in AF, similar considerations apply to venous thromboembolism. Projects are under way in the UK Academic Health Science Networks to deploy the combination of routine genotyping and self-testing to confirm the efficacy of more effective targeting of warfarin. Meanwhile, the NHS expenditure on anticoagulation is climbing steeply. DOACs account for $31 \%$ of treated patients but around 93\% of expenditure on anticoagulants. Uptake of the new drugs varies widely, ranging from $8 \%$ to $>60 \%$ in different Clinical Commissioning Groups across Englandi. As new warfarin patients decline and DOAC share of the market increases, expenditure will rise steeply despite the lack of evidence of efficacy in routine practice. Even allowing for the initial cost of genotyping of around $£ 50$, routine monitoring, typically $£ 150$ per annum which can include the depreciated $£ 300$ cost for self-testing devices on a lease arrangement, warfarin offers major savings. Warfarin costs an average of $£ 0.83$ per month, whereas the average monthly cost of DOACs is listed at just $>£ 50$. Thus, overall NHS annual expenditure could be reduced by $>£ 0.5 \mathrm{~B}$ per annum in the near future without impairment of the nation's health if DOACs are restricted to those of working age and/or are shown to be sensitive to warfarin.

Acknowledgements MP thanks the MRC Centre for Drug Safety Science for infrastructure support. MP is also supported by the NIHR CLAHRC North West Coast.

Funding No external funding was needed apart from the analysis of anticoagulant adherence. This was commissioned by Roche Diagnostics and donated to the authors for inclusion, with the knowledge of the original analysts.

Competing interests None declared.

Provenance and peer review Not commissioned; externally peer reviewed.

Data sharing statement No additional data are available.

Open Access This is an Open Access article distributed in accordance with the terms of the Creative Commons Attribution (CC BY 4.0) license, which permits others to distribute, remix, adapt and build upon this work, for commercial use, provided the original work is properly cited. See: http://creativecommons.org/licenses/by/4.0/

(C) Article author(s) (or their employer(s) unless otherwise stated in the text of the article) 2018. All rights reserved. No commercial use is permitted unless otherwise expressly granted.

\section{REFERENCES}

1. AFA. Atrial fibrillation association. 2017. http://www. heartrhythmalliance.org/afa/uk/af-related-stroke

2. Homma S, Thompson JL, Pullicino PM, et al. Warfarin and aspirin in patients with heart failure and sinus rhythm. N Engl J Med 2012;366:1859-69. 
3. Mant J, Hobbs FD, Fletcher K, et al. Warfarin versus aspirin for stroke prevention in an elderly community population with atrial fibrillation (the Birmingham Atrial Fibrillation Treatment of the Aged Study, BAFTA): a randomised controlled trial. Lancet 2007;370:493-503.

4. McDonald MG, Rieder MJ, Nakano M, et al. CYP4F2 is a vitamin K1 oxidase: An explanation for altered warfarin dose in carriers of the V433M variant. Mol Pharmacol 2009;75:1337-46.

5. Odén A, Fahlén M, Hart RG. Optimal INR for prevention of stroke and death in atrial fibrillation: a critical appraisal. Thromb Res 2006;117:493-9.

6. Hylek EM, Skates SJ, Sheehan MA, et al. An analysis of the lowest effective intensity of prophylactic anticoagulation for patients with nonrheumatic atrial fibrillation. N Engl J Med 1996;335:540-6.

7. Kirkwood TB. Calibration of reference thromboplastins and standardisation of the prothrombin time ratio. Thromb Haemost 1983;49:238-44.

8. Pirmohamed $\mathrm{M}$, et al. Adverse drug reactions as cause of admission to hospital: prospective analysis of 18820 patients. BMJ 2004;329:15-19.

9. Patel MR, Mahaffey KW, Garg J, et al. Rivaroxaban versus warfarin in nonvalvular atrial fibrillation. N Engl J Med 2011;365:883-91.

10. Granger CB, Alexander JH, McMurray JJ, et al. Apixaban versus warfarin in patients with atrial fibrillation. $N$ Engl J Med 2011;365:981-92.

11. Connolly SJ, Ezekowitz MD, Yusuf S, et al. Dabigatran versus warfarin in patients with atrial fibrillation. $N$ Engl J Med 2009;361:1139-51.

12. Heidbuchel $\mathrm{H}$, Verhamme $\mathrm{P}$, Alings $\mathrm{M}$, et al. Updated European heart rhythm association practical guide on the use of non-vitamin $\mathrm{K}$ antagonist anticoagulants in patients with non-valvular atrial fibrillation. Europace 2015;17:1467-507.

13. Kmietowicz $Z$. Boehringer Ingelheim withheld safety analyses on new anticoagulant, The BMJ investigation finds. BMJ 2014;349:g4756.

14. Pollack CV, Reilly PA, Eikelboom J, et al. Idarucizumab for Dabigatran Reversal. N Engl J Med 2015;373:511-20.

15. Siegal DM, Curnutte JT, Connolly SJ, et al. Andexanet alfa for the reversal of factor Xa inhibitor activity. N Engl J Med 2015;373:2413-24.

16. Cohen D. Data on trial of anticoagulant is to be reanalyzed after discovery that investigators used faulty device. BMJ 2015;351:h6431.
17. Ruff CT, Giugliano RP, Braunwald E, et al. Comparison of the efficacy and safety of new oral anticoagulants with warfarin in patients with atrial fibrillation: a meta-analysis of randomised trials. Lancet 2014;383:955-62.

18. Abraham NS, Singh S, Alexander GC, et al. Comparative risk of gastrointestinal bleeding with dabigatran, rivaroxaban, and warfarin: population based cohort study. BMJ 2015;350:h1857.

19. Jackevicius CA, Tsadok MA, Essebag V, et al. Early non-persistence with dabigatran and rivaroxaban in patients with atrial fibrillation. Heart 2017:103:1331-8.

20. Douxfils J, Buckinx F, Mullier F, et al. Dabigatran etexilate and risk of myocardial infarction, other cardiovascular events, major bleeding, and all-cause mortality: a systematic review and meta-analysis of randomized controlled trials. J Am Heart Assoc 2014;3:e000515

21. Petzold T, Thienel M, Konrad I, et al. Oral thrombin inhibitor aggravates platelet adhesion and aggregation during arterial thrombosis. Sci Trans/ Med 2016;8:367ra168.

22. Stolk LM, de Vries F, Ebbelaar C, et al. Risk of myocardial infarction in patients with atrial fibrillation using vitamin $\mathrm{K}$ antagonists, aspirin or direct acting oral anticoagulants. Br J Clin Pharmacol 2017;83:1835-43.

23. Loffredo L, Perri L, Del Ben M, et al. New oral anticoagulants for the treatment of acute venous thromboembolism: are they safer than vitamin $\mathrm{K}$ antagonists? A meta-analysis of the interventional trials. Intern Emerg Med 2015;10:499-506.

24. Haaland GS, Falk RS, Straume O, et al. Association of warfarin use with lower overall cancer incidence among patients older than 50 years. JAMA Intern Med 2017;177:1774-80.

25. Aithal GP, Day CP, Kesteven PJ, et al. Association of polymorphisms in the cytochrome P450 CYP2C9 with warfarin dose requirement and risk of bleeding complications. Lancet 1999;353:717-9.

26. Rieder MJ, Reiner AP, Gage BF, et al. Effect of VKORC1 haplotypes on transcriptional regulation and warfarin dose. $N$ Engl J Med 2005;352:2285-93.

27. Pirmohamed M, Burnside G, Eriksson N, et al. A randomized trial of genotype-guided dosing of warfarin. $N$ Engl J Med 2013;369:2294-303.

28. Mega JL, Walker JR, Ruff CT, et al. Genetics and the clinical response to warfarin and edoxaban: findings from the randomised, double-blind ENGAGE AF-TIMI 48 trial. Lancet 2015;385:2280-7. 


\section{Correction: Direct oral anticoagulants versus warfarin: is new always better than the old?}

Burn J, Pirmohamed M. Direct oral anticoagulants versus warfarin: is new always better than the old? Open Heart 2018;5:e000712. doi: 10.1136/openhrt-2017-000712

The competing interests statement was erroneously missed from this article when it first published.

Professor Sir John Burn is Professor of Clinical Genetics at Newcastle University and a non-executive director of NHS England where at the time of submission he was deputy chair of Specialised Commissioning at NHS England. He helped write the 2003 government White Paper on Genetics which used warfarin genotyping as an example of future deployment of Pharmacogenetics. He has been working for 10 years with a small biotechnology company called QuantuMDx Ltd, based in Newcastle, to develop point of care DNA diagnostic devices. He is now employed part time as medical director and Chair and hold shares in the company. He chose warfarin genotyping as a suitable target for development of a disposable diagnostic cassette for use in clinic. This is expected to enter trials in the next 12 months.

Professor Sir Munir Pirmohamed's work on warfarin pharmacogenetics has been funded via a number of sources including Department of Health, NIHR, EU Commission and the NW Coast AHSN. The grants were awarded to the University of Liverpool. LGC, an international company, were partners in the EU-PACT Consortium, an FP7 funded EU project, and produced the point-of-care genotyping platform that was used in the EU-PACT trial. They have also provided their genotyping platform and reagents free of charge for the implementation project on warfarin pharmacogenetics which is on-going in the NW Coast (which is co-funded by the NIHR NW Coast CLAHRC and NW Coast AHSN). Professor Pirmohamed does not hold any shares in LGC or any other company marketing genotyping tests for warfarin. He also do not hold any shares in companies marketing warfarin or any of the DOACs. He is a Commissioner on Human Medicines and Chair its Pharmacovigilance Expert Advisory Group - these are independent committees which provide advice to the MHRA on the safety, efficacy and quality of medicines in the UK.

Open Access This is an Open Access article distributed in accordance with the terms of the Creative Commons Attribution (CC BY 4.0) license, which permits others to distribute, remix, adapt and build upon this work, for commercial use, provided the original work is properly cited. See: http://creativecommons.org/licenses/by/4.0/

Open Heart 2018;5:e000712corr1. doi:10.1136/openhrt-2017-000712corr1

Check for updates 